\title{
PGA $\times$ BSA: A Measure of Psoriasis Severity Tested in Patients with Active Psoriatic Arthritis and Treated with Certolizumab Pegol
}

\author{
Jessica A. Walsh, Terri Arledge, Tommi Nurminen, Luke Peterson, and Jeffrey Stark
}

\begin{abstract}
Objective. The product of physician's global assessment and body surface area (PGA $\times \mathrm{BSA}$ ) to assess psoriasis severity has previously been investigated in patients with psoriasis, with the aim of assessing PGA $\times$ BSA as an alternative to the time-consuming Psoriasis Area and Severity Index (PASI). Here, we investigate $\mathrm{PGA} \times \mathrm{BSA}$ as an alternative to PASI in patients with psoriatic arthritis (PsA).

Methods. Analyses used data from the double-blind, placebo-controlled, RAPID-PsA trial (NCT01087788) that investigated the efficacy of certolizumab pegol (CZP) in patients with PsA. Outcomes assessed whether the PGA $\times$ BSA and PASI results were comparable, and whether these outcomes correlated with one another or with the Dermatology Life Quality Index (DLQI).

Results. For CZP-treated patients, both PGA $\times$ BSA and PASI demonstrated similar sensitivities to treatment between baseline and Week 24, with mean improvements of $77.4 \%$ and $69.0 \%$, respectively. Similar improvements were also seen with placebo (PGA×BSA: $3.2 \%$, PASI: $6.1 \%$ ). Achievement of $75 \%$ response criterion in PGA $\times \mathrm{BSA}$ and PASI was attained by similar proportions of patients with CZP (PGA $\times$ BSA75: 59.0\%, PASI75: 61.4\%) and placebo (PGA $\times$ BSA75: 15.1\%, PASI75: $15.1 \%)$. Cross tabulations showed high concordance between achievement of response outcomes in PGA $\times$ BSA and PASI (79.6-95.2\%). Spearman correlations revealed strong correlations between PGA $\times$ BSA and PASI at baseline $(r=0.78 ; n=225)$ and percentage improvement to Week 24 $(\mathrm{r}=0.85 ; \mathrm{n}=186)$. Both outcomes were only moderately correlated with DLQI $(\mathrm{r}=0.41-0.50$; $\mathrm{n}=179-249)$.

Conclusion. PGA $\times$ BSA is sensitive to changes in skin manifestations in patients with PsA treated with CZP. Further, PGA $\times$ BSA correlates strongly with PASI, and achievement of $75 \%$ improvement was similar for PGA×BSA and PASI. (First Release May 1 2018; J Rheumatol 2018:45;922-8; doi:10.3899/jrheum.170244)
\end{abstract}

Key Indexing Terms:

PSORIASIS

Psoriasis is a genetic, immune-mediated disease, affecting $1-3 \%$ of the US population. About $30 \%$ of patients with psoriasis are also affected by psoriatic arthritis (PsA) ${ }^{1}$, which is associated with a wide variety of additional symptoms that contribute to the disease burden. Factors such as joint pain, erosive joint damage, enthesitis, and dactylitis, as well as psoriasis of the skin and nails further increase the longterm

From the Division of Rheumatology, University of Utah, Salt Lake City, Utah; UCB Pharma, Raleigh, North Carolina; UCB Pharma, Smyrna, Georgia, USA; UCB Pharma, Monheim, Germany.

This study was funded by UCB Pharma. All costs associated with the development of this manuscript were funded by UCB Pharma.

$T A, T N, L P$, and JS are employees of UCB Pharma.

J.A. Walsh, MD, Division of Rheumatology, University of Utah; T. Arledge, DVM, UCB Pharma; T. Nurminen, MSc, UCB Pharma; L. Peterson, MSc, UCB Pharma; J. Stark, MD, UCB Pharma.

Address correspondence to Dr. J.A. Walsh, Division of Rheumatology, University of Utah School of Medicine, 30 N 1900 E, Salt Lake City, Utah 84132,USA.E-mail: Jessica.Walsh@hsc.utah.edu

Full Release Article. For details see Reprints and Permissions at jrheum.org

Accepted for publication December 14, 2017.

\section{PSORIATIC ARTHRITIS}

effect on patients' quality of life, physical function, and ability to work ${ }^{2,3}$.

The wide variety of symptoms makes evaluation of overall disease activity and response to therapy difficult in PsA; however, accurate assessment is essential for the clinician to determine the most appropriate treatment. These assessment difficulties are particularly true of skin disease because of limitations of the currently used outcome measures.

The Psoriasis Area and Severity Index (PASI) is the most widely used tool for the measurement of skin involvement and is considered the "gold standard" for clinical trials ${ }^{4,5}$. However, PASI assessments are complex and time-consuming, and can be insensitive in patients with milder forms of psoriasis ${ }^{5,6}$. The US Food and Drug Administration (FDA) does not accept PASI as a standalone efficacy endpoint in clinical trials for psoriasis. Rather, the FDA often requires the inclusion of a physician's global assessment (PGA) of psoriasis as a coprimary efficacy endpoint ${ }^{7,8}$. However, PGA instruments do not consider the amount of coverage of skin involvement; they assess only the plaque qualities ${ }^{9}$. Therefore, 2 patients with similar plaque morphology but

Personal non-commercial use only. The Journal of Rheumatology Copyright $\odot$ 2018. All rights reserved 
with different amounts of surface involvement (e.g., $30 \%$ vs $3 \%$ ) could have the same PGA score.

To address these limitations, an alternative outcome measure has been proposed: the product of PGA and body surface area (PGA $\times \mathrm{BSA})$, which has the advantages of being simpler than the PASI and giving meaningful scores regardless of psoriasis severity ${ }^{10}$. The PGA $\times$ BSA tool is simpler than the PASI because skin disease severity and psoriasis BSA are scored only once for the entire body, rather than 4 times for each of the 4 body regions measured by the $\mathrm{PASI}^{9}$. Additionally, the PGA $\times \mathrm{BSA}$ calculation is straightforward, whereas PASI scoring requires multiple mathematical computations. The Group for Research and Assessment of Psoriasis and Psoriatic Arthritis has recently highlighted the need for measures that are both simple and valid to assess clinical responses of psoriasis. These measures must be accessible to healthcare providers, patients, regulators, and payers. Importantly, these easily understood measures also have the potential to enhance the dialogue between physician and patient, and improve the measurement of disease activity over time ${ }^{11}$.

Although simpler than PASI, it must be demonstrated that the PGA $\times \mathrm{BSA}$ is a comparable outcome measure. In patients with psoriasis, $\mathrm{PGA} \times \mathrm{BSA}$ has been shown to correlate strongly with PASI ${ }^{10}$, with recent findings suggesting this simpler alternative tool may be used to assess disease severity and response to therapy in patients with moderate to severe psoriasis ${ }^{12}$.

The analyses reported here aimed to investigate whether PGA $\times$ BSA can be used as an alternative to PASI for measuring psoriasis severity and response to therapy in patients with PsA using data from the RAPID-PsA trial.

\section{MATERIALS AND METHODS}

Patients. Analyses were carried out using data from the phase III RAPID-PsA multicenter clinical trial in Europe, North America, and Latin America (NCT01087788) ${ }^{13}$ that investigated the efficacy of certolizumab pegol (CZP), a PEGylated, Fc-free anti-tumor necrosis factor (TNF) agent, compared to placebo in patients with active PsA. The study was approved by a national, regional, or independent ethics committee or institutional review board at participating sites (ethical approval on behalf of the first author of this publication was provided by The University of Utah Institutional Review Board). The study was conducted in accordance with applicable regulatory and International Conference on Harmonisation Good Clinical Practice requirements, based on the Declaration of Helsinki and local laws. All patients provided written informed consent prior to any protocol-specific procedures being performed. Data from 73 sites were included in this posthoc analysis, including 26 from North America, 22 from Central/Eastern Europe, 14 from Western Europe, and 11 from Latin America. The study was double-blind to Week 24, dose-blind to Week 48, then open-label to Week 216. At Week 0, patients were randomized 1:1:1 to placebo, CZP $200 \mathrm{mg}$ every 2 weeks (Q2W) or CZP $400 \mathrm{mg}$ every 4 weeks (Q4W), administered subcutaneously. Placebo patients who did not achieve a 10\% improvement from baseline in both swollen and tender joints at Weeks 14 and 16 underwent mandatory escape and were rerandomized at Week 16 to 1 of the 2 active treatment arms, in a blinded manner. The analyses presented here used data from the initial 24-week double-blind, placebo-controlled, period of RAPID-PsA.

To participate in RAPID-PsA, patients were required to fulfill the
ClASsification criteria for Psoriatic ARthritis ${ }^{14}$ and to have active disease, defined as $\geq 3$ tender joints and $\geq 3$ swollen joints, and either erythrocyte sedimentation rate $\geq 28 \mathrm{~mm} / \mathrm{h}$ or C-reactive protein $>7.9 \mathrm{mg} / \mathrm{l}$, with duration $\geq 6$ months (full eligibility criteria have been previously published) ${ }^{13}$. Patients must have failed treatment with, or have been intolerant to, $\geq 1$ disease-modifying antirheumatic drug. The primary clinical ${ }^{13}$ and radiographic $^{15}$ endpoints of RAPID-PsA have been reported elsewhere.

Outcomes assessed. Skin outcomes included in these analyses were assessed in all patients with $\geq 3 \%$ BSA affected by psoriasis at baseline. Outcomes assessed included the PASI (scored on a 0-72 scale), PGA (scored on a 0-5 scale, where $0=$ clear, $1=$ almost clear, $2=$ mild, $3=$ moderate, $4=$ moderate to severe, and $5=$ severe), BSA (scored as the percentage body area affected by psoriasis; 0-100\%), the Dermatology Life Quality Index (DLQI; scored on a $0-30$ scale), and the PGA $\times$ BSA [0-500; derived posthoc by multiplying patients' PGA scores $(0-5)$ by patients' psoriasis BSA (0-100\%)]. Achievement of $\geq 75 \%$ response (improvement from baseline) was also considered for PASI (PASI75) and PGA $\times$ BSA (PGA $\times$ BSA75)

Statistical analyses. Data are reported for patients randomized to CZP (combined dose regimens: $200 \mathrm{mg} \mathrm{Q} 2 \mathrm{~W}$ and $400 \mathrm{mg} \mathrm{Q} 4 \mathrm{~W}$ ) or placebo. Outcomes are also reported separated by the severity of patients' skin involvement at baseline (PASI $\geq 10$ vs PASI $<10$, with PASI $\geq 10$ indicating severe skin involvement).

Data are reported as observed case or with imputation. Last observation carried forward imputation was used for continuous outcomes measures and nonresponder imputation for dichotomous outcomes. For placebo patients who underwent mandatory escape at Week 16 to active treatment, their Week 16 response was carried forward to Week 24 .

Responsiveness to change was measured between baseline and Week 24 with ANCOVA-adjusted standardized effect sizes, based on the difference in change from baseline between treatments divided by the residual SD, with treatment groups as an explanatory factor and baseline values as a covariate.

The level of agreement between PASI75 and PGA×BSA75 responses at Week 24 was assessed using cross tabulations. Similar analyses were performed for PASI90 and PGA×BSA90, and PASI100 and PGA $\times$ BSA100 responses.

Spearman correlation coefficients were calculated to compare PGA $\times$ BSA, PASI, and DLQI.

\section{RESULTS}

Baseline characteristics. Of 409 patients randomized in RAPID-PsA, 252 had $\geq 3 \%$ BSA at baseline (166 received CZP and 86 placebo). Of these patients, 98 had PASI $\geq 10$ at baseline (70 received CZP and 28 placebo) and 153 PASI $<10$ (95 received CZP and 58 placebo). There were more males in the PASI $\geq 10$ group $(60.2 \%)$ compared to the PASI $<10$ group $(36.6 \%)$. Joint inflammation was similar in patients with PASI $\geq 10$ and PASI $<10$ at baseline, though all measures of psoriatic skin involvement were higher in patients with PASI $\geq 10$ (Table 1).

PASI and PGA $\times B S A$ responses following anti-TNF treatment. At Week 24, the mean percentage improvement from baseline was similar for PGA $\times$ BSA and PASI in patients receiving CZP (PGA×BSA: 77.4\%; PASI: 69.0\%) or placebo (PGA $\times$ BSA $3.2 \%$; PASI: 6.1\%; Figure 1A). The standardized effect sizes, adjusted for baseline values, of the CZP treatment group versus the placebo group at Week 24 were also similar for the 2 measures: $-1.0(95 \% \mathrm{CI}-1.28$ to -0.73$)$ for PASI and $-1.05(-1.36$ to -0.74$)$ for PGA $\times$ BSA. Greater responses were observed in patients with PASI $\geq 10$ than

Personal non-commercial use only. The Journal of Rheumatology Copyright $\subset$ 2018. All rights reserved. 
Table 1. Baseline characteristics for patients from the RAPID-PsA trial with $\geq 3 \%$ psoriasis BSA at baseline and PASI $\geq 10$ or $<10$. Values are mean \pm SD unless otherwise specified.

\begin{tabular}{|c|c|c|c|}
\hline Characteristics & $\begin{array}{l}\geq 3 \% \text { Psoriasis BSA } \\
\text { at Baseline, } \mathrm{n}=252\end{array}$ & $\begin{array}{c}\geq 3 \% \text { Psoriasis BSA } \\
\text { and PASI } \geq 10 \text { at } \\
\text { Baseline, } \mathrm{n}=98\end{array}$ & $\begin{array}{c}\geq 3 \% \text { Psoriasis BSA } \\
\text { and PASI }<10 \\
\text { at Baseline, } \mathrm{n}=153\end{array}$ \\
\hline \multicolumn{4}{|l|}{ Demographics } \\
\hline Age, yrs & $47.8 \pm 11.5$ & $48.1 \pm 11.7$ & $47.6 \pm 11.4$ \\
\hline Female, $\%$ & 54.0 & 39.8 & 63.4 \\
\hline Weight, kg & $85.7 \pm 18.8$ & $86.6 \pm 19.4$ & $84.9 \pm 18.4$ \\
\hline \multicolumn{4}{|l|}{ Prior medication, $\%$} \\
\hline 1 prior nonbiologic DMARD & 61.9 & 59.2 & 63.4 \\
\hline$\geq 2$ prior nonbiologic DMARD & 36.1 & 37.8 & 35.3 \\
\hline Prior anti-TNF & 22.2 & 27.6 & 19.0 \\
\hline \multicolumn{4}{|l|}{ Disease characteristics } \\
\hline Tender joint count, 0-68 & $21.6 \pm 15.1$ & $23.4 \pm 15.4$ & $20.6 \pm 14.9$ \\
\hline Swollen joint count, 0-66 & $11.2 \pm 8.3$ & $12.7 \pm 9.8$ & $10.3 \pm 7.0$ \\
\hline HAQ-DI, range $0-3$ & $1.4 \pm 0.6$ & $1.4 \pm 0.6$ & $1.4 \pm 0.6$ \\
\hline Psoriasis BSA, \% & $23.4 \pm 22.2$ & $39.9 \pm 23.7$ & $12.9 \pm 13.0$ \\
\hline PASI & $11.7 \pm 11.9, \mathrm{n}=251$ & $22.8 \pm 12.1, n=98$ & $4.5 \pm 2.6, n=153$ \\
\hline $\mathrm{PGA} \times \mathrm{BSA}$ & $79.4 \pm 92.3, \mathrm{n}=226$ & $150.6 \pm 105.2, \mathrm{n}=88$ & $34.1 \pm 40.2, n=137$ \\
\hline DLQI & $10.8 \pm 7.3, \mathrm{n}=250$ & $14.5 \pm 7.3, \mathrm{n}=98$ & $8.4 \pm 6.2, \mathrm{n}=151$ \\
\hline mNAPSI $^{1}$ & $3.6 \pm 2.1, \mathrm{n}=202$ & $3.9 \pm 2.1, n=83$ & $3.4 \pm 2.1, \mathrm{n}=119$ \\
\hline
\end{tabular}

${ }^{1}$ For patients with nail disease at baseline. PsA: psoriatic arthritis; BSA: body surface area; PASI: Psoriasis Area and Severity Index; DMARD: disease-modifying antirheumatic drugs; TNF: tumor necrosis factor; HAQ-DI: Health Assessment Questionnaire-Disability Index; PGA: physician's global assessment; DLQI: Dermatology Life Quality Index; mNAPSI: modified Nail Psoriasis Area Severity Index.

PASI $<10$ at baseline (Figure 1B). However, within both subgroups (PASI $\geq 10$ and PASI $<10$ ), the responses were similar between the 2 outcome measures. At Week 24, CZP-treated patients with PASI $\geq 10$ at baseline showed mean percentage improvements of $88.5 \%$ (PGA $\times \mathrm{BSA}$ ), and $82.2 \%$ (PASI), while patients with PASI $<10$ at baseline showed improvements of $68.7 \%$ (PGA $\times$ BSA) and $60.0 \%$ (PASI). This was also reflected in the effect sizes: in the subgroup of patients with milder psoriasis at baseline (PASI $<10)$, adjusted standardized effect sizes were -0.69 (95\% CI -1.03 to -0.35 ) for PASI and $-0.72(95 \% \mathrm{CI}-1.09$ to -0.34$)$ for PGA $\times$ BSA. In the subgroup of patients with PASI $\geq 10$, adjusted standardized effect sizes were -1.64 (95\% CI -2.13 to -1.14$)$ for PASI and $-1.76(95 \% \mathrm{CI}-2.34$ to -1.18$)$ for PGA $\times$ BSA.

At Week 24, a comparable proportion of CZP-treated patients achieved a PGA $\times$ BSA75 and a PASI75 response (59.0\% and $61.4 \%$, respectively; Figure 2A). Similarly, patients receiving placebo achieved PASI75 and PGA $\times$ BSA75 responses in comparable proportions (both $15.1 \%$ ).

As expected, a greater proportion of CZP-treated patients with PASI $\geq 10$ at baseline achieved PGA $\times$ BSA75 and PASI75 at Week 24 than those with PASI $<10$ at baseline. Again, however, within subgroups, similar proportions of CZP-treated patients achieved $75 \%$ response criteria in PGA $\times$ BSA and PASI (PGA $\times$ BSA75 achieved by $68.6 \%$ of patients with PASI $\geq 10$, compared to $52.6 \%$ of patients with PASI $<10$; PASI75 achieved by $77.5 \%$ of patients with PASI $\geq 10$, compared to $49.5 \%$ of patients with PASI $<10$ ).
Response rates for the 2 outcome measures were also comparable in placebo-treated patients (Figure 2B).

Concordance of PGA $\times B S A$ and PASI responses. Further analyses considered whether patients achieving/not achieving a PGA $\times$ BSA75 response also achieved/did not achieve a PASI75 response. The majority of patients $(83.9 \%)$ had agreement in their responses to both outcomes (Table 2A).

The number of patients achieving a $75 \%$ response in 1 outcome but not the other was similar between the 2 outcomes, with $9.7 \%$ of patients achieving a PGA×BSA75 response but not a PASI75 response, and $6.5 \%$ of patients achieving PASI75 but not PGA $\times$ BSA75. This demonstrates the comparability of the outcomes. High correlations between the outcomes were seen when cross tabulations considered $90 \%$ or $100 \%$ responses (Table $2 \mathrm{~B}$ and Table $2 \mathrm{C}$ ).

Spearman correlations (Figure 3 ) revealed strong correlations between actual values PGA $\times$ BSA and PASI at baseline (0.78), and percentage changes from baseline to Week 24 (0.85), which were similar across patients from North America, Latin America, Western Europe, and Eastern Europe (data not shown).

In terms of percentage change from baseline, $\mathrm{PGA} \times \mathrm{BSA}$ also correlated more strongly with PASI than did BSA alone (PASI correlation with BSA: 0.77), and slightly more strongly than with PGA alone (PASI correlation with PGA: 0.83). However, both PGA $\times$ BSA and PASI were only moderately correlated with DLQI at baseline ( 0.46 and 0.50 , respectively) and at Week 24 (percentage change from baseline, both 0.41).

Personal non-commercial use only. The Journal of Rheumatology Copyright $\odot$ 2018. All rights reserved. 
A

CZP

PBO

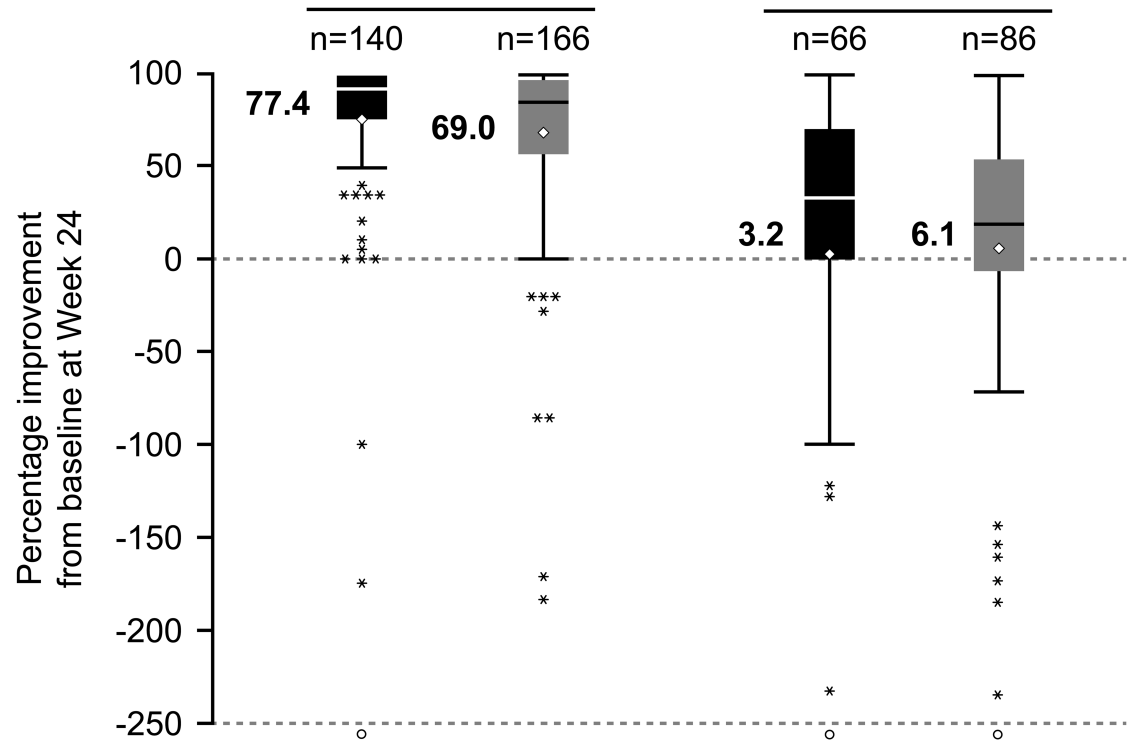

B
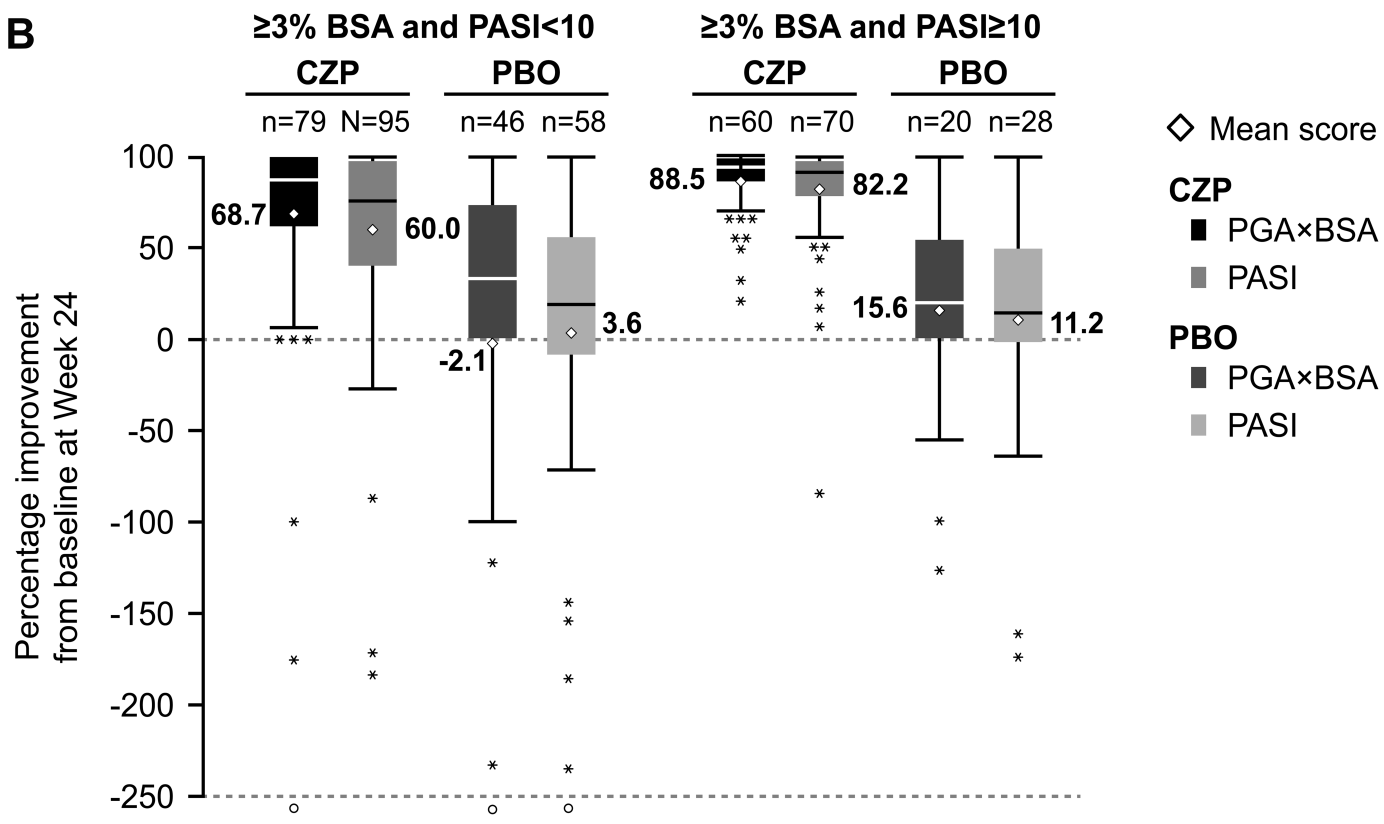

100

$\mathrm{n}=79 \mathrm{~N}=95 \quad \mathrm{n}=46 \mathrm{n}=58$

Mean score

- PGA $B$ BSA

PASI

Figure 1. Percentage improvements from baseline to Week 24 in PGA $\times$ BSA and PASI (LOCF). A. All patients with $\geq 3 \%$ psoriasis BSA at baseline. B. Patients with $\geq 3 \%$ psoriasis BSA at baseline and PASI $\geq 10$ or PASI $<10$. Extreme observations not shown in the following: PGA $\times$ BSA (CZP -300, PBO -1233); PASI (PBO -331). CZP: certolizumab pegol; PBO: placebo; PGA $\times$ BSA: physician's global assessment $\times$ body surface area; PASI: Psoriasis Area and Severity Index; LOCF: last observation carried forward.

\section{DISCUSSION}

We demonstrated that the PGA $\times$ BSA can be used as an alternative outcome measure to PASI for measuring disease activity and treatment response in skin manifestations in patients with PsA. PGA $\times$ BSA is an outcome measure comparable to PASI and is sensitive to change in skin manifestations of PsA with varying levels of skin severity at baseline (PASI $\geq 10$ and PASI < 10). Achievement of $75 \%$ improvement in patients with PsA at Week 24 was comparable for PGA $\times$ BSA and PASI, with similar effect sizes observed for the 2 measures, when adjusting for baseline values.

Although PGA $\times$ BSA was found to correlate strongly with PASI, both outcomes were only moderately correlated with DLQI. This may reflect the fact that both PGA $\times$ BSA and PASI measure only plaque quality and surface area and do not account for other factors affecting cutaneous psoriasis

Personal non-commercial use only. The Journal of Rheumatology Copyright (c) 2018. All rights reserved. 
A

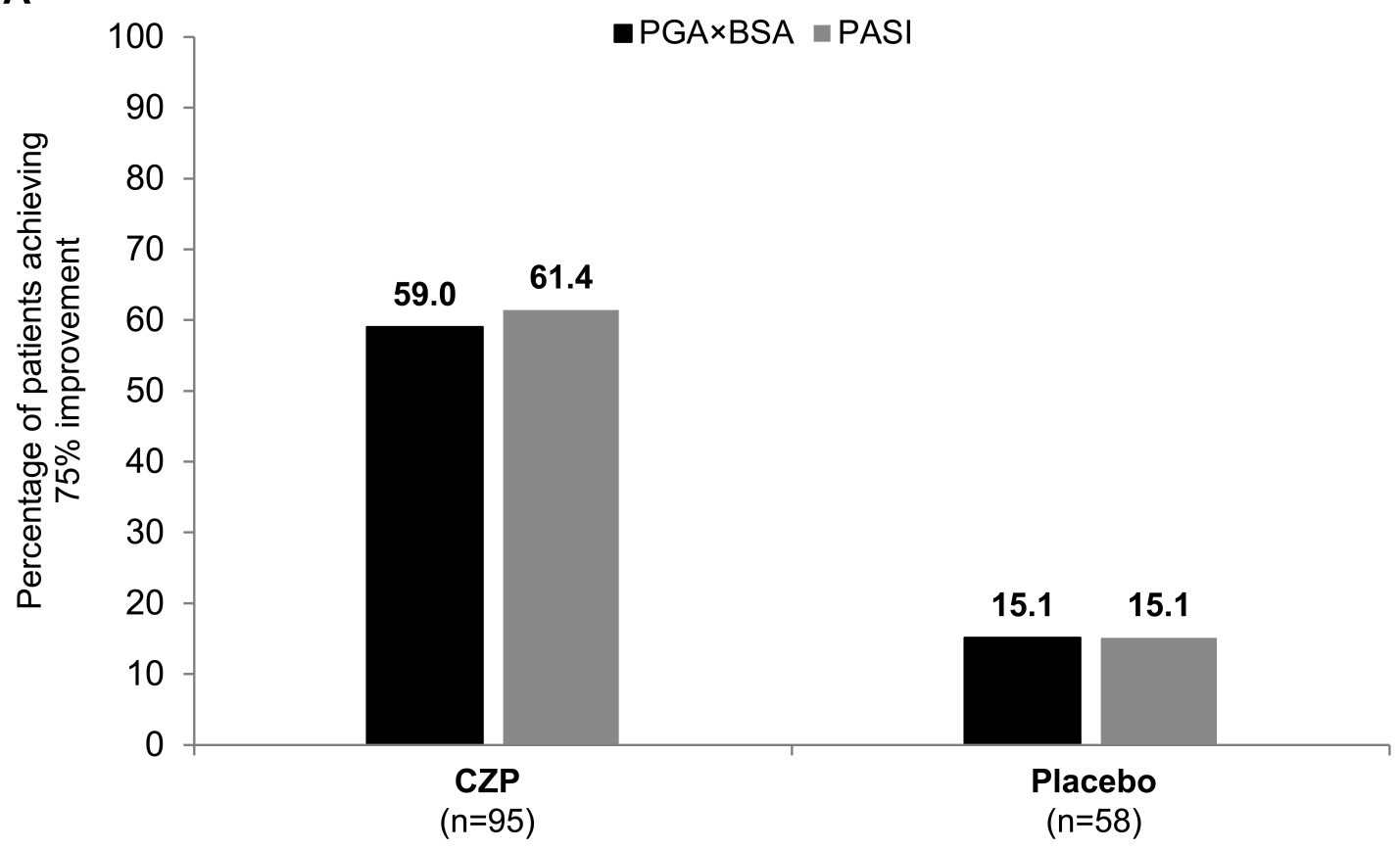

B

-PGA×BSA $=\mathrm{PASI}$

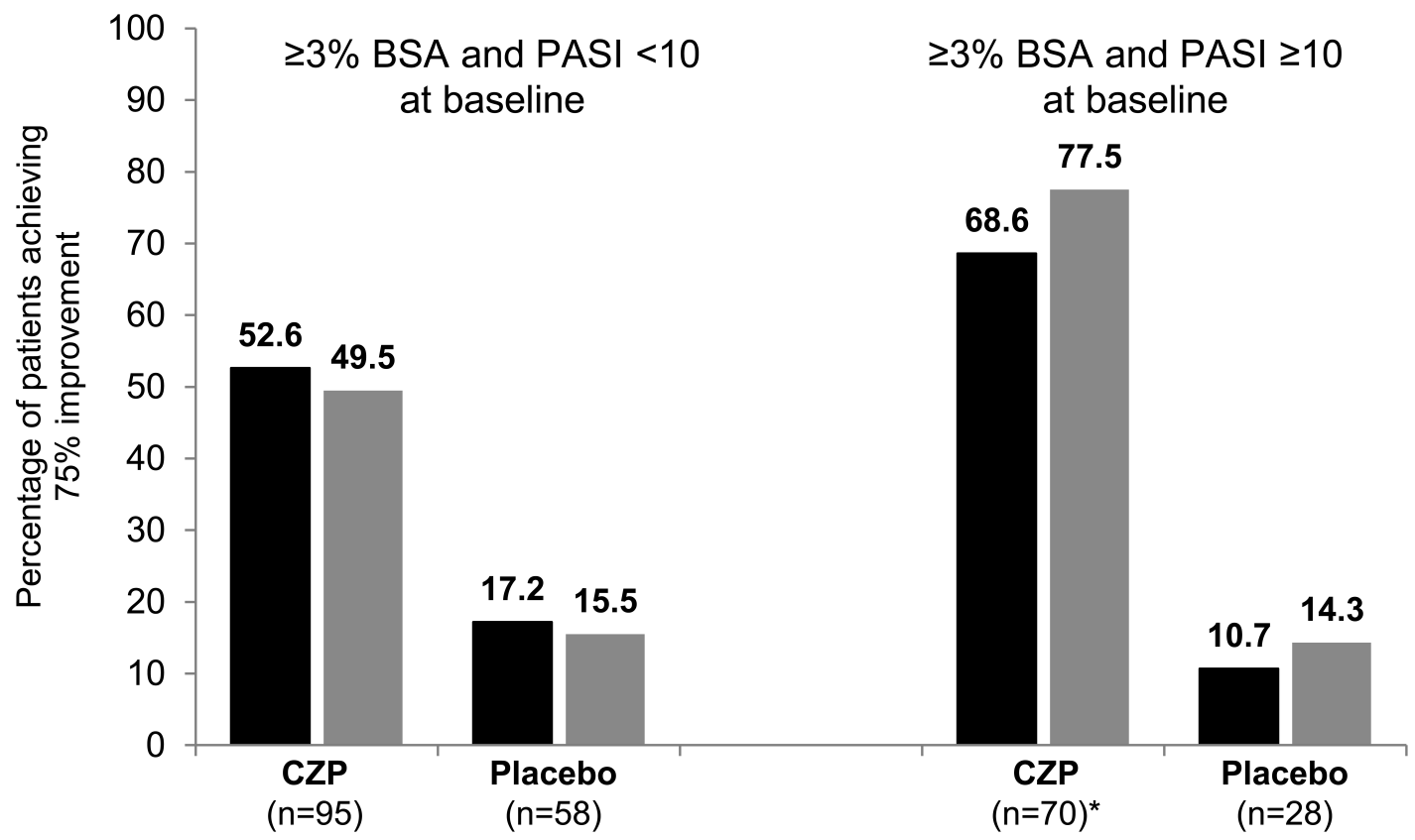

Figure 2. Percentage of patients achieving a 75\% response in PASI (PASI75) or PGA $\times$ BSA (PGA $\times$ BSA75) at Week 24 (NRI). A. All patients with $\geq 3 \%$ psoriasis BSA at baseline. B. Patients with $\geq 3 \%$ psoriasis BSA at baseline and PASI $\geq 10$ or PASI $<$ 10. *For CZP patients with PASI responses and severe skin involvement at baseline, $\mathrm{n}=71$. For placebo patients who escaped early, their response at Week 16 was used from the time the escape medication was initiated. PASI: Psoriasis Area and Severity Index; PGA×BSA: physician's global assessment $\times$ body surface area; NRI: nonresponder imputation.

disease severity, such as itch, visibility, or discomfort, which are important factors affecting patients' quality of life.

The PGA×BSA has previously been evaluated in patients with psoriasis, both through retrospective analyses of registry data ${ }^{10,16}$ and recently in a posthoc analysis using data from the ESTEEM 1 and ESTEEM 2 trials $^{12}$. Consistent with our 
Table 2A. Cross-tabulations of PGA $\times$ BSA $75 \%$ and PASI 75 response achievement at Week 24 (observed case data; all patients with $\geq 3 \%$ psoriasis BSA at baseline).

\begin{tabular}{lcccc}
\hline & & \multicolumn{2}{c}{ PGA $\times$ BSA75 } & Total \\
\hline \multirow{2}{*}{ PASI75 } & Yes & No & $114(61.3)$ \\
& Yes & $102(54.8)$ & $\mathbf{1 2}(\mathbf{6 . 5})$ & $72(38.7)$ \\
& No & $\mathbf{1 8}(\mathbf{9 . 7 )}$ & $54(29.0)$ & \\
& Total & $120(64.5)$ & $66(35.5)$ & \\
& &
\end{tabular}

Shaded numbers $=$ agreement in responses: $83.8 \%$. Bold face numbers $=$ disagreement in responses: $16.2 \%$. Sensitivity of PGA $\times$ BSA75 relative to PASI $75=89.5 \%$, specificity $=75.0 \%$, diagnostic OR $=25.5$.

Table $2 B$. Cross-tabulations of PGA×BSA 95\% and PASI 90 response achievement at Week 24 (observed case data; all patients with $\geq 3 \%$ psoriasis BSA at baseline).

\begin{tabular}{lcccc}
\hline & & PGA & & \\
& & Yes & No & Total \\
\hline \multirow{2}{*}{ PASI90 } & Yes & $63(33.9)$ & $\mathbf{1 1 ( 5 . 9 )}$ & $74(39.8)$ \\
& No & $\mathbf{2 7 ( 1 4 . 5 )}$ & $85(45.7)$ & $112(60.2)$ \\
& Total & $90(48.4)$ & $96(51.6)$ & \\
\hline
\end{tabular}

Shaded numbers $=$ agreement in responses: $79.6 \%$. Bold face numbers $=$ disagreement in responses: $20.4 \%$. Sensitivity of PGA $\times$ BSA90 relative to PASI $90=85.1 \%$, specificity $=75.9 \%$, diagnostic $\mathrm{OR}=18.0$.

Table 2C. Cross-tabulations of PGA×BSA 100\% and PASI 100 response achievement at Week 24 (observed case data; all patients with $\geq 3 \%$ psoriasis BSA at baseline)

\begin{tabular}{lcccc}
\hline & & \multicolumn{3}{c}{ PGA $\times$ BSA100 } \\
PASI100 & Yes & No & Total \\
& Yes & $36(19.4)$ & $\mathbf{3}(\mathbf{1 . 6})$ & $39(21.0)$ \\
& No & $\mathbf{6 ( 3 . 2 )}$ & $141(75.8)$ & $147(79.0)$ \\
& Total & $42(22.6)$ & $144(77.4)$ & \\
\hline
\end{tabular}

Shaded numbers $=$ agreement in responses: $95.2 \%$. Bold face numbers = disagreement in responses: $4.8 \%$. Sensitivity of PGA $\times$ BSA100 relative to PASI100 $=92.3 \%$, specificity $=95.9 \%$, diagnostic OR $=282.0$. PGA $\times$ BSA: physician's global assessment $\times$ body surface area; PASI: Psoriasis Area and Severity Index.

data, the ESTEEM analyses showed that PGA $\times$ BSA and PASI were highly correlated with each other both at baseline and at Week 16 of treatment. While there were some differences between the patient populations included in the ESTEEM trials and our analysis, notably with a greater psoriasis severity in ESTEEM (PASI $\geq 12, \mathrm{BSA} \geq 10 \%$, PGA $\geq 3$ at baseline), the similar findings suggest $\mathrm{PGA} \times \mathrm{BSA}$ to be a consistent and simple measure for the assessment of psoriasis across different conditions and at varying levels of disease severity.

Here, the product of PGA $\times$ BSA correlated more strongly with PASI than either component alone, although for PGA this was a fairly small incremental improvement. Despite this relative similarity at a population level, within individual patients $\mathrm{PGA} \times \mathrm{BSA}$ can provide a more representative overview of their disease by taking into account both the plaque qualities and extent of psoriasis. Use of either component alone could lead to misclassification, for example, by categorizing a patient with a PGA of 5 and a
BSA of $0.2 \%$ as having more severe disease than a patient with a PGA of 2 and a BSA of $50 \%$.

One limitation of our study is that both the PGA $\times$ BSA and PASI were assessed at the same visits by the same physician. The physician's scoring of these outcomes may therefore not be completely independent of the other and thus these analyses may overestimate the agreement between the 2 outcomes. It should also be noted that we used PGA scored on a $0-5$ scale; however, other available versions of this instrument use different scales and may affect the reproducibility of the results seen here. In the ESTEEM analyses, the correlations between PASI and PGA×BSA were lower than those seen here, which may be in part because of the different PGA scale used. Additionally, standardized effect sizes should be interpreted with caution because both PASI and PGA $\times$ BSA scores after treatment are skewed toward the lower end of the respective scales, which may affect calculations of effect size. Further research is therefore required from prospective studies to further validate this tool.

Personal non-commercial use only. The Journal of Rheumatology Copyright $\subset$ 2018. All rights reserved. 


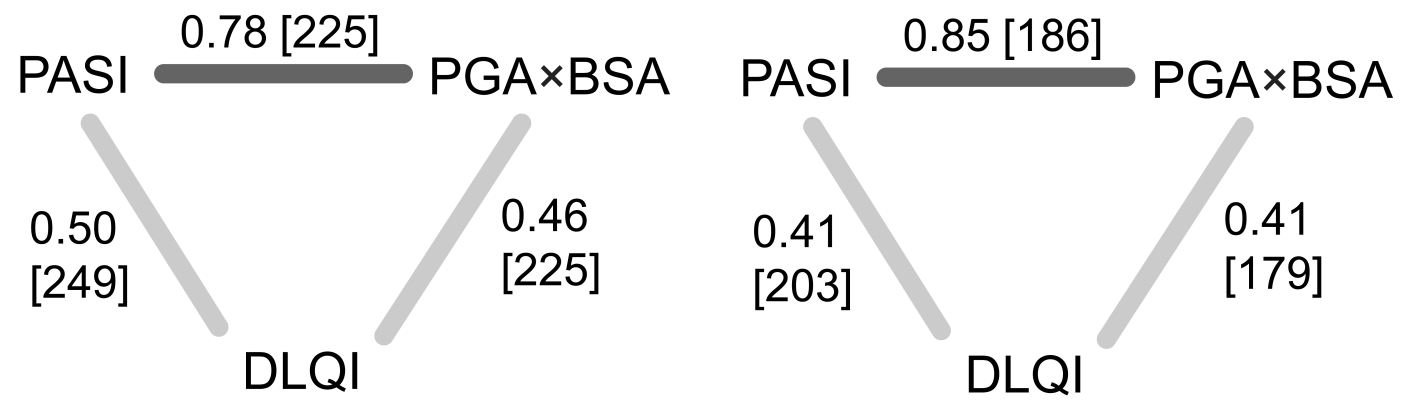

\begin{abstract}
*Spearman's rank correlation coefficient interpretation:
\end{abstract}

\section{$0 \quad 0.25$}

No correlation
PASI $0.85[186]$ PGSA

\section{DLQI}

\subsection{1}

Figure 3. Spearman correlations between PGA $\times$ BSA, PASI, and DLQI (observed case data; all patients with $\geq 3 \%$ psoriasis BSA at baseline). Numbers shown are Spearman's rank correlation (no. patients). PASI: Psoriasis Area and Severity Index; PGA×BSA: physician's global assessment $\times$ body surface area; DLQI: Dermatology Life Quality Index.

In patients with PsA, PGA $\times$ BSA may be considered a practical alternative to PASI for the measurement of psoriasis severity and response to therapy.

\section{ACKNOWLEDGMENT}

The authors thank the patients and their caregivers, in addition to the investigators and their teams who contributed to this study, as well as to the Marriott Daughters Foundation and the Ben B. and Iris M. Margolis Foundation. The authors also acknowledge Irfan Khan, UCB Pharma, Smyrna, Georgia, USA, for publication coordination, and Helen Chambers, DPhil, from Costello Medical Consulting, Cambridge, UK, for medical writing and editorial assistance in preparing this manuscript for publication, based on the authors' input and direction.

\section{REFERENCES}

1. Gladman D, Antoni C, Mease P, Clegg D, Nash P. Psoriatic arthritis: epidemiology, clinical features, course, and outcome. Ann Rheum Dis 2005;64:ii14-7.

2. Salaffi F, Carotti M, Gasparini S, Intorcia M, Grassi W. The health-related quality of life in rheumatoid arthritis, ankylosing spondylitis, and psoriatic arthritis: a comparison with a selected sample of healthy people. Health Qual Life Outcomes 2009;7:25.

3. Tillett W, de-Vries C, McHugh NJ. Work disability in psoriatic arthritis: a systematic review. Rheumatology 2012;51:275-83.

4. Schmitt J, Wozel G. The psoriasis area and severity index is the adequate criterion to define severity in chronic plaque-type psoriasis. Dermatology 2005;210:194-9.

5. Armstrong AW, Parsi K, Schupp CW, Mease PJ, Duffin KC. Standardizing training for psoriasis measures: effectiveness of an online training video on Psoriasis Area and Severity Index assessment by physician and patient raters. JAMA Dermatol 2013;149:577-82.

6. Youn SW, Choi CW, Kim BR, Chae JB. Reduction of inter-rater and intra-rater variability in psoriasis area and severity index assessment by photographic training. Annals Dermatol 2015;27:557-62.

7. Naldi L. Scoring and monitoring the severity of psoriasis. What is the preferred method? What is the ideal method? Is PASI passé? facts and controversies. Clin Dermatol 2010;28:67-72.
8. Robinson A, Kardos M, Kimball AB. Physician Global Assessment (PGA) and Psoriasis Area and Severity Index (PASI): why do both? A systematic analysis of randomized controlled trials of biologic agents for moderate to severe plaque psoriasis. J Am Acad Dermatol 2012;66:369-75.

9. Bonifati C, Berardesca E. Clinical outcome measures of psoriasis. Reumatismo 2007;59 Suppl 1:64-7.

10. Walsh JA, McFadden M, Woodcock J, Clegg DO, Helliwell P, Dommasch E, et al. Product of the physician global assessment and body surface area: a simple static measure of psoriasis severity in a longitudinal cohort. J Am Acad Dermatol 2013;69:931-7.

11. Merola JF, Gottlieb AB. Practical assessment of psoriasis clinical severity in both clinical trials and clinical practice settings: a report from the GRAPPA 2016 annual meeting. J Rheumatol 2017; 44:691-2.

12. Duffin KC, Papp KA, Bagel J, Levi E, Chen R, Gottlieb AB. Evaluation of the physician global assessment and body surface area composite tool for assessing psoriasis response to apremilast therapy: results from ESTEEM 1 and ESTEEM 2. J Drugs Dermatol 2017; $16: 147-53$.

13. Mease P, Fleischmann R, Deodhar AA, Wollenhaupt J, Khraishi M, Kielar D, et al. Effect of certolizumab pegol on signs and symptoms in patients with psoriatic arthritis: 24-week results of a Phase 3 double-blind randomised placebo-controlled study (RAPID-PsA). Ann Rheum Dis 2014;73:48-55.

14. Taylor W, Gladman D, Helliwell P, Marchesoni A, Mease P, Mielants H; CASPAR Study Group. Classification criteria for psoriatic arthritis: development of new criteria from a large international study. Arthritis Rheum 2006;54:2665-73.

15. van der Heijde D, Fleischmann R, Wollenhaupt J, Deodhar A, Kielar $\mathrm{D}$, Woltering F, et al. Effect of different imputation approaches on the evaluation of radiographic progression in patients with psoriatic arthritis: results of the RAPID-PsA 24-week phase III double-blind randomised placebo-controlled study of certolizumab pegol. Ann Rheum Dis 2014;73:233-7.

16. Chiesa Fuxench ZC, Callis Duffin K, Siegel M, Van Voorhees AS, Gelfand JM. Validity of the simple-measure for assessing psoriasis activity (S-MAPA) for objectively evaluating disease severity in patients with plaque psoriasis. J Am Acad Dermatol 2015; 73:868-70. 